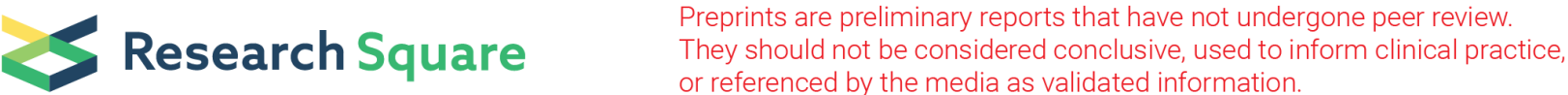

\section{Canagliflozin Attenuates Lipotoxicity in Cardiomyocytes and Protects Diabetic Mouse Hearts by Targeting the mTOR/HIF-1a Pathway}

\section{Pengbo Sun}

Tsinghua University https://orcid.org/0000-0002-7326-8847

Yipei Ding

Tsinghua University

Jingyi Luo

Tsinghua University

Jin Zhong

Tsinghua University

Weidong Xie ( $\nabla$ xiewd@sz.tsinghua.edu.cn )

Tsinghua University

Original investigation

Keywords: Canagliflozin, heart failure, lipotoxicity, mTOR, HIF-1a

Posted Date: December 14th, 2020

DOI: https://doi.org/10.21203/rs.3.rs-123774/v1

License: (c) (1) This work is licensed under a Creative Commons Attribution 4.0 International License. Read Full License 


\section{Abstract}

\section{Background}

Lipotoxicity plays an important role in the development of diabetic cardiomyopathy and heart failure (HF). Canagliflozin (CAN), a marketed sodium-glucose co-transporter 2 inhibitor, has significant beneficial effects on HF. However, the potential pharmacological mechanism is still unknown.

\section{Methods}

In this study, we evaluated the protective effects and mechanism of CAN in the hearts of a C57BL/6J diabetic mouse model induced by a high-fat diet/streptozotocin (HFD/STZ) for 12 weeks in vivo and using HL-1 cells (a type of mouse cardiomyocyte line) induced by palmitic acid (PA) in vitro.

\section{Results}

CAN could significantly alleviate lipid accumulation and inflammatory responses in the hearts of the HFD/STZ-induced diabetic mice. Furthermore, CAN significantly attenuated the inflammatory injury induced by PA in the HL-1 cells. In addition, CAN bound to the mammalian target of rapamycin (mTOR) and significantly inhibited mTOR phosphorylation and hypoxia inducible factor-1a (HIF-1a) expression.

\section{Conclusion}

CAN attenuated lipotoxicity in cardiomyocytes and protected diabetic mouse hearts by targeting the mTOR/HIF-1 a pathway.

\section{Background}

Diabetes is a chronic metabolic disorder, characterized by hyperglycemia and complications caused by insulin deficiency and/or resistance. The cardiovascular complications are a major cause of mortality and morbidity in diabetic patients. Diabetic patients have an increased risk of cardiovascular mortality by $86 \%$ and $185 \%$ are hospitalization due to heart failure (HF) compared with these factors in non-diabetic patients (1). In the absence of overt myocardial ischemia and hypertension, diabetes independently causes changes in the myocardial structure and function, which is known as diabetic cardiomyopathy (2). Diabetic cardiomyopathy is one of the important causes for the development of HF. However, the potential pathological mechanisms of diabetic cardiomyopathy remain unclear.

A healthy heart requires a large amount of ATP to maintain normal function, and approximately $70 \%$ ATP is produced by fatty acid oxidation (FAO) (3). However, in diabetic hearts, mitochondrial dysfunction leads to fatty acid metabolic disorder, and increased lipid accumulation and oxidation, which lead to increased oxidative stress, inflammation, and lipotoxicity (4). Previous research also showed that increased myocardial triglyceride content in diabetic patients was associated with impaired left ventricular diastolic function (5). 
Inhibiting lipotoxicity in the hearts of diabetic patients is a potential strategy for the treatment of diabetic cardiomyopathy and subsequent HF. However, currently there is not a suitable drug to attenuate lipotoxicity in the hearts of diabetic patients. CAN is a sodium-glucose co-transporter 2 (SGLT2) inhibitor and is an antidiabetic drug. Clinical research found it has extra beneficial effects by decreasing hospitalization and mortality rates of HF in T2DM patients (6). Furthermore, some studies have indicated that CAN may exert its cardioprotective effect independent of its hypoglycemic activity (7). CAN also improved lipid metabolism and attenuated the development of non-alcoholic fatty liver disease (8). However, whether CAN regulated lipid metabolism and attenuated lipotoxicity in the hearts of diabetic patients remains unclear.

mTOR and hypoxia inducible factor-1a (HIF-1a) are important factors mediating the pathological progression of HF in diabetes patients $(9,10)$. mTOR, as an energy metabolism factor, was also found to regulate HIF-1a expression (11). However, no suitable drugs have been found to target the mTOR-HIF-1a pathway or to treat diabetic cardiomyopathy or HF. In this study, we aimed to evaluate the protective effects of CAN in a high-fat diet/streptozotocin (HFD/STZ)-induced diabetic HF mouse model and in palmitic acid (PA)-induced HL-1 cell lipotoxicity, and investigate whether CAN targeted the mTOR-HIF-1a pathway to exert a cardioprotective effect.

\section{Material And Methods}

\subsection{HFD/STZ-induced diabetic mouse models}

C57BL/ 6 mice (body weight, 18 \pm 2 g; 4-weeks-old; male) were purchased from Guangzhou Medical Animal Centre (Guangzhou, China) and housed under controlled conditions (constant temperature: $22 \pm 2^{\circ} \mathrm{C}$; constant humidity: $60 \pm 5 \% ; 12 \mathrm{~h}$ dark/light cycle). The study was performed in strict accordance with the National Institutes of Health Guide for the Care and Use of Laboratory Animals and the protocol was approved by the Bioethics Committee of Shenzhen International Graduate School, Tsinghua University, China (Ethics issue [2020] No. 9). The diabetic mouse models were fed with a HFD (41\% energy from fat; Beijing HFK Bioscience, Beijing, China, H10141). The models were intraperitoneally injected with STZ (40 mg/kg; Sangon Biotech Co., Ltd., Shanghai, China), which was freshly dissolved in ice-cold $0.1 \mathrm{M}$ citrate solution ( $\mathrm{pH} 4.5$ ), according to a previous study (12). After one week, the diabetic mice were divided into two groups: An untreated HFD/STZ control group and a CAN (ChenXi Biotech, Shanghai, China)-treated group ( $25 \mathrm{mg} / \mathrm{kg} / \mathrm{d}$; dissolved in $0.5 \%$ sodium salt of carboxymethyl cellulose [CMC-Na; Sangon Biotech, Shanghai, China]). The dose of CAN administered to the mice was converted according to the clinical dose in humans $(100 \mathrm{mg} / \mathrm{kg} /$ day). The normal control and HFD/STZ control groups were orally administered with an equal volume of $0.5 \%$ CMC-Na daily. The body weight of the mice, and food and water uptake was monitored once a week. After 12 weeks of treatment, the mice were subjected to fasting for $6 \mathrm{~h}$, anesthetized with urethane, which was dissolved in saline (intraperitoneal injection of urethane at a dose of $1000 \mathrm{mg} \mathrm{kg}-1$; Sangon Biotech Co., Ltd., Shanghai, China), then sacrificed while under anesthesia. The blood was collected from the orbital plexus veins, and the serum was extracted from the blood samples using centrifugation (1000 rpm for $10 \mathrm{~min}$ at $4^{\circ} \mathrm{C}$ ) and stored at 
$-20^{\circ} \mathrm{C}$ for further analysis. Simultaneously, unrecovered anesthetized animals were sacrificed using cervical dislocation by skillful well-trained investigators, and the hearts and abdominal adipose tissues were removed and weighed. A portion of the heart tissue was immersed in $4 \%$ paraformaldehyde solution for regular pathological slicing, and hematoxylin and eosin (H\&E) and Masson staining. The remaining samples were instantly frozen by using liquid nitrogen and stored at $-80^{\circ} \mathrm{C}$ for further analysis.

\subsection{Biochemical analysis in animals}

The levels of serum cholesterol, triglyceride, glucose, lactate dehydrogenase (LDH) and creatine kinase (CK) were measured using regular commercial kits (BioSino Bio-Technology \& Science Inc, Beijing, China). B-type natriuretic peptide (BNP) was detected using an ELISA kit (YingXinBio, Shanghai, China). Total protein from the heart tissue was extracted using a cell lysis buffer, and the protein concentration was determined with a commercial kit (both from Beyotime Institute of Biotechnology, Shanghai, China). The cholesterol, triglyceride, and glycogen levels in the heart tissues were analyzed with commercial kits (Nanjing Jiancheng, Nanjing, China) and their levels were normalized to the protein concentration.

\subsection{Cell culture}

The HL-1 cells were purchased from Fenghui Biotechnology Company, (Changsha, China) and cultured in high glucose DMEM (Gibco ${ }^{\circledR}$; Thermo Fisher Scientific, Inc., USA) supplemented with $10 \%$ premium fetal bovine serum (Pan Biotech, Germany) and 1\% penicillin-streptomycin antibiotic (Gibco ${ }^{\mathrm{Tm}}$; Thermo Fisher Scientific, Inc., USA), at $37^{\circ} \mathrm{C}$ in a humidified atmosphere with $5 \% \mathrm{O}_{2}$. The cells were seeded at a density of either $8 \times 10^{3}$ cells per well in 96 -well plates, $2 \times 10^{4}$ cells per well in 24 -well plates, or $3 \times 10^{5}$ cells in 6 well plates (all from Guangzhou Jet Bio-Filtration Co., Ltd.). The HL-1 cells were incubated with PA $(0.25$ $\mathrm{mM}$; Sigma-Aldrich, USA) for $24 \mathrm{~h}$. Different concentrations of CAN $(0.625,1.25$ and $2.5 \mu \mathrm{g} / \mathrm{ml})$, rapamycin (RAPA; 100, 200, and $400 \mathrm{nM}$; MedChemExpress, USA), and LW6 (5, 10 and $20 \mu \mathrm{M}$; MedChemExpress, USA) were added to investigate the anti-inflammatory activities on PA-induced HL-1 cells. PA was dissolved in 3\% bovine serum albumin (BSA; Biofroxx, Germany) solution. CAN, RAPA, and LW6 were dissolved in dimethyl sulfoxide (DMSO; Beyotime Institute of Biotechnology, Shanghai, China). Blank control cells were treated with an equal volume of BSA or DMSO solution. After $24 \mathrm{~h}$ of treatment, oil red $O$ (Sangon Biotech Co., Ltd., Shanghai, China) staining was performed to observe fat accumulation. Total protein and RNA was extracted, as described previously (13). The mRNA expression levels of Hif-1a, II-1a, II-6, and Tnf-a were analyzed using reverse transcription-quantitative PCR (RT-qPCR). The phosphorylation of HIF-1a and mTOR was measured using western blot analysis.

\subsection{MTT assay}

MTT (Sangon Biotech, Shanghai, China) was dissolved in phosphate buffer solution (PBS; $0.01 \mathrm{M}$; $\mathrm{pH}, 7.0$ ) at a concentration of $5 \mathrm{mg} / \mathrm{ml}$ and sterilized by filtration with $0.22 \mu \mathrm{m}$ filters. The HL- 1 cells ( $8 \times$ $10^{3}$ per well) were seeded into 96-well plates. The CAN, RAPA and LW6 reagents were dissolved in DMSO. The HL-1 cells were incubated with PA $(0.25 \mathrm{mM})$ for $24 \mathrm{~h}$ to induce lipotoxicity. Different concentrations 
of CAN $(0.625,1.25$ and $2.5 \mu \mathrm{g} / \mathrm{ml})$, RAPA $(100,200$ and $400 \mathrm{nM})$, and LW6 $(5,10$ and $20 \mu \mathrm{M})$ were simultaneously added for $24 \mathrm{~h}$ to investigate the protective activity on PA-induced lipotoxicity. The blank or untreated control groups were treated with an equal volume of BSA solution. After $24 \mathrm{~h}$ of treatment, $20 \mu \mathrm{l}$ MTT solution was added to each well and incubated for $4 \mathrm{~h}$ in the incubator. Following which, the cell medium was removed and $200 \mu \mathrm{LMSO}$ was added to dissolve the purple formazan crystals in each well. Optical density values at $490 \mathrm{~nm}$ (OD490) were analyzed using a microplate spectrophotometer. The following equation was used to determine the percentage cell viability: Cell viability $\%=($ OD 490 of each group/average OD490 of the blank control group) $\times 100 \%$.

\subsection{RT-qPCR assay}

The mRNA expression level was analyzed, as described in a previous study (14). Approximately $500 \mathrm{ng}$ total RNA was reverse transcribed into cDNA using the Evo M-MLV RT Premix for the qPCR kit (Accurate Biotechnology, Hunan, China). RT-qPCR was performed using SYBR Green Premix Pro Taq HS qPCR kit (Accurate Biotechnology, Hunan, China). The primers were synthesized by Genewiz, Inc., Suzhou, China (Table 1).

\subsection{Western blot analysis}

Western blot analysis was conducted according to a previous study, with a slight modification (14). The cell lysates were collected, and the protein samples were separated using 10\% SDS-PAGE, then transferred to PVDF membranes (Pall, USA). Subsequently, the membranes were blocked with blocking buffer ( $5 \% \mathrm{~g} / \mathrm{ml} \mathrm{skimmed} \mathrm{milk} \mathrm{power} \mathrm{(Anchor,} \mathrm{New} \mathrm{Zealand),} \mathrm{which} \mathrm{was} \mathrm{dissolved} \mathrm{in} \mathrm{PBS} \mathrm{containing}$ 0.5\% g/ml Tween-20 (PBST; Sangon Biotech Co., Ltd., Shanghai, China) for $1 \mathrm{~h}$, then incubated with primary antibodies dissolved in $3 \% \mathrm{BSA}$ overnight at $4^{\circ} \mathrm{C}$. After the membrane was washed with PBST buffer (PBS added with 0.5\% Tween-20) three times, the secondary antibodies (dissolved in blocking buffer) were added and incubated for $1 \mathrm{~h}$. After washing again, the protein bands were visualized using enhanced chemiluminescence (Pierce ${ }^{\text {Tw }}$ ECL Western Blotting Substrate; Thermo Fisher Scientific, Inc., USA). The following primary antibodies were used: $\beta$-actin (mouse; 1:5000; A1978; Sigma-Aldrich), HIF-1a (mouse; 1:1000; 14179S; Cell Signaling Technology, Inc.), mTOR (mouse; 1:1000; 2983; Cell Signal Technology, Inc.), and phosphorylated (p)-mTOR (mouse; 1:1000; 5536; Cell Signal Technology Inc.).

\subsection{ROS assay}

The fluorescent probe, 2', 7'-dichlorodihydrofluorescein diacetate (Beyotime Institute of Biotechnology) was used to detect reactive oxygen species (ROS) levels, as described in our previous study (13). The HL1 cells $\left(2 \times 10^{4}\right.$ per well) were seeded into 24 -well plates, then incubated with PA $(0.25 \mathrm{mM})$ for $24 \mathrm{~h}$ to induce ROS generation. CAN $(2.5 \mu \mathrm{g} / \mathrm{ml})$ was simultaneously treated for $24 \mathrm{~h}$ to investigate the activity against ROS. The blank and untreated control cells were treated with an equal volume of DMSO or BSA solution. The fluorescent signal was measured using a fluorescence microscope (excitation wavelength of $485 \mathrm{~nm}$; emission wavelength of $525 \mathrm{~nm}$ ). 


\subsection{Immunofluorescence and confocal assay}

The immunofluorescence assay in the HL-1 cells was performed as previously described (13). First, circular transparent glass slides (cat. no. 12-545-83; Fisherbrand ${ }^{\text {TI' }}$ microscope cover glass; Thermo Fisher Scientific, Inc. USA) were placed at the bottom of a 6-well plate. The cells, at a density of $2.5 \times 105 /$ well, were added to the surface of glass slides in a 6-well plate, then cultured in fresh medium. After $24 \mathrm{~h}$ of culture, the cells were divided into three groups: Normal untreated control group, PA ( $0.25 \mathrm{mM})$ - treated group, and PA $(0.25 \mathrm{Mm})$ - and CAN $(2.5 \mu \mathrm{g} / \mathrm{ml})$ - treated group. After $24 \mathrm{~h}$, the immobilized cells on the slides were washed with PBS, then fixed with $4 \%$ paraformaldehyde (Sangon Biotech, Shanghai, China) in PBS for 15 mins. The cells were washed with PBS three times, then incubated with $0.1 \%$ Triton X-100 in PBS for 15 min. After three washes with PBS, the cells were blocked with 3\% BSA (Sangon Biotech, Shanghai, China) in PBS for $1 \mathrm{~h}$. After blocking, the cells were incubated with p-mTOR (Ser2448) rabbit monoclonal antibody mAb (1:200; 5536S; Cell Signaling Technology, Inc., USA) and HIF-1 a mouse monoclonal antibody (1:200; CST14179S; Cell Signaling Technology, Inc., USA) in 3\% BSA for $1 \mathrm{~h}$, then washed with PBS three times. Next, the cells were incubated with goat anti-rabbit IgG H\&L (1:200; ab150077; Alexa Fluor ${ }^{\circledR} 488 ;$ Abcam, UK) or goat anti-mouse IgG H\&L (1:100; ab150119; Alexa Fluor $^{\circledR}$ 647 ; Abcam, UK) in 3\% BSA for $1 \mathrm{~h}$. The fluorescence in the cell was observed using confocal microscopy (Olympus, Corporation Japan) and analyzed using FV10-ASW Viewer v3.1 and ImageJ software.

\subsection{Apoptosis assays}

For the flow cytometry assays, the HL-1 cells $\left(2 \times 10^{5} /\right.$ well) were cultured in 6-well plate. The cells were divided into three groups: Normal untreated control, PA $(0.25 \mathrm{mM})$ - treated, and PA (0.25 Mm)- and CAN $(2.5 \mathrm{\mu g} / \mathrm{ml})$-treated groups. After $24 \mathrm{~h}$ of treatment, the collected cells were fluorescently stained using the $\mathrm{YF}^{\circledR}$ 488-Annexin V and PI Apoptosis commercial kit (Y6002; US Everbright Inc., Suzhou, Jiangsu, China). Flow cytometry was detected using a BD Accuri $\mathrm{C} 6$ flow cytometer (BD Biosciences, USA) and the results were analyzed using FlowJo v10.6.2 software.

For the confocal assay, the HL-1 cells $\left(2 \times 10^{5} /\right.$ well) were seeded onto the surface of circular transparent glass slides in a 6-well plate and analyzed according to the confocal assay protocol, as described above. The cells on the slide were fluorescently stained using the $\mathrm{YF}^{\circledR}$ 488-Annexin V and PI Apoptosis commercial kit (Y6002; US Everbright Inc, Suzhou, Jiangsu, China).

\subsection{Pathway enrichment analyze assay}

SwissTargetPrediction (http://www.swisstargetprediction.ch/) is a web server for small molecule drug target prediction (15). Using the web server, we obtained possible targets of CAN. ClueGo is a powerful tool for network analysis, annotation, and visualization. The predicted targets were analyzed using ClueGo 2.5.4 to enrich the pathway network for the Kyoto Encyclopedia of Genes and Genomes pathway database (16) (the threshold was set at $P<0.05)$. GeneCards is a database containing details of diseaserelated genes and proteins (16). The key words "diabetes" and "HF" were used in GeneCards to search for 
disease-related genes. From the results of the diabetes- and HF-related genes, we selected the top 200 genes and analyzed them using ClueGo (the threshold was set at $\mathrm{P}<0.001$ ). The enrichment results and intersection analysis were conducted using Origin Pro 2018c.

\subsection{Molecular docking assay}

SwissDock is an online sever for small molecule-protein docking (17). The mTOR crystal structure was obtained using the Protein Data Bank (PDB ID: 4DRI). The molecular docking model and affinity energy of CAN and RAPA with mTOR were analyzed using SwissDock (www.swissdock.ch) and the results were visualized using the software, Chimera v1.14.

\subsection{Affinity chromatography}

Affinity chromatography was conducted according to a previous study (18) with a slight modification.

Preparation of the drug: CAN ( $5 \mathrm{mg}$ ) was dissolved in $0.35 \mathrm{ml}$ of $80 \%$ ethanol, while sucrose $(3 \mathrm{mg})$ was dissolved in $0.35 \mathrm{ml}$ of $40 \%$ ethanol. The two solutions were dropped into $0.25 \mathrm{ml}$ of $8 \mathrm{mg} / \mathrm{ml}$ sodium periodate (Sangon Biotech Co., Ltd.) solution and left for $1 \mathrm{~h}$ for the reaction to occur. Following which, $0.5 \mathrm{ml}$ of $100 \mathrm{mM}$ sodium carbonate buffer $(\mathrm{pH}, 9)$ was added to the two solutions.

Preparation of the affinity chromatography column: Two $5 \mathrm{ml}$ centrifuge chromatography tubes (Thermo Fisher Scientific, Inc., USA) were prepared, and $1 \mathrm{ml}$ resin (Carboxylink ${ }^{\text {T"M }}$ Coupling Gel; Thermo Fisher Scientific, Inc., USA) was transferred into one of the tubes. The supernatants were removed using centrifugation at $1000 \mathrm{rpm}$ for $20 \mathrm{~s}$ and washed with deionized water three times.

Linking the drug onto the column: $0.375 \mathrm{ml}$ of $4 \mathrm{mg} / \mathrm{ml}$ sodium borohydride solution (Sangon Biotech Co., Ltd.) was added into the aforementioned two resin tubes, mixed in a shaker, and left to react for $4 \mathrm{~h}$. The cover of the tube was opened every half an hour to release the gas generated in the reaction.

Affinity chromatography: The HL-1 cells were cultured in a 10-cm plate. The cell lysates were collected and loaded into the prepared chromatography resin tubes and incubated overnight at $4^{\circ} \mathrm{C}$. The proteins bound to the resin was separated using SDS-PAGE and identified using Coomassie brilliant blue staining and western blot analysis.

\subsection{Statistical analysis}

All the data are presented as the mean \pm SD. Unpaired t-test (two tailed) was used when comparing two groups and GraphPad Prism v8 software. One-way ANOVA with Tukey's post hoc test was used for multiple comparisons and SPSS v22 software. $P<0.05$ was considered to indicate a statistically significant difference.

\section{Results}




\subsection{The beneficial effects of CAN on body/adipose weight, blood lipid/glucose level and heart lipid/glucose accumulation in HFD/STZ-induced diabetic mice}

An unhealthy life style has caused a significant increase in the morbidity and mortality rates in patients with diabetic complications, and the excessive intake of saturated fatty acids is one of the strongest risk factors in diabetic cardiovascular disease. Multiple mechanisms have been associated with the development of diabetic cardiovascular complications, such as oxidative stress, inflammation and cell apoptosis (19). In this study, we first used HFD/STZ to induce a type 2 diabetic HF model in the C57BL/6J mice and these were treated with CAN ( $25 \mathrm{mg} / \mathrm{kg} /$ day) for 12 weeks. During the experiment, the body weight of the HFD/STZ diabetic group was significantly increased at 1, 2, 5, 8, 9, and 11 weeks compared with that in the normal control group (Fig. 1a). However, CAN significantly inhibited this increase at 3-12 weeks compared with that in the HFD/STZ diabetic control group. We also monitored the diet and water intake of the different groups (Fig. 1b and 1C). The energy/water uptake in the CANtreated HFD/STZ diabetic group demonstrated a slightly increased energy intake compared with that in the HFD/STZ diabetic control group. However, the HFD/STZ diabetic group showed a significant increase in the abdominal adipose tissue index (abdominal adipose tissue weight/body weight) compared with that in the normal control group and this increase was significantly inhibited by CAN (Fig. 1d). Therefore, the body and adipose tissue weights reduced by CAN were not associated with the inhibition of dietary energy intake.

Subsequently, we evaluated the effects of CAN on glucose and lipid levels in the blood serum of the HFD/STZ diabetic HF model. The concentrations of triglycerides, cholesterol, and glucose in the serum showed a significant increase in the HFD/STZ diabetic group compared with that in the normal control group. However, CAN significantly attenuated this increase in the HFD/STZ-induced diabetic group (Fig. 1e-g). In addition, we evaluated the effects of CAN on glucose and lipid levels in the heart tissue. The increased concentrations of triglycerides, cholesterol, and glycogen in the heart tissues of the HFD/STZ diabetic group were also significantly inhibited by CAN (Fig. 1h-j). However, these beneficial effects of CAN on glucose and lipid metabolism in the serum and heart tissue were not associated with the regulation of dietary intake.

\subsection{The protective effect of CAN on HF and metabolic inflammation induced by HFD/STZ in vivo}

The progression of $\mathrm{HF}$ is accompanied by cardiomyocyte remodeling and fibrosis. Left ventricular hypertrophy and blunt heart apical are two notable morphological features (20). The heart morphological features in the HFD/STZ group were changed: Left ventricular hypertrophy developed, the ventricular wall thickened, and the heart apical became blunt compared with that in the normal group; however, the heart shape was improved in the CAN group compared with that in the HFD/STZ control group after 12 weeks of treatment (Fig. 2a). We calculated the area of the cell nucleus; the HFD/STZ group had a bigger cell nucleus compared with that in the normal control group (Fig. 2b), while CAN significantly reduced this area. This finding indicated that CAN inhibited myocardial hypertrophy in vivo. We also observed fibrosis in the cardiomyocytes in the different groups using Masson staining. Compared with that in the normal 
control group, the cardiomyocytes in the HFD/STZ group were arranged in a disorderly manner and accompanied with notable fibrosis; however, these pathological changes were also reversed after CAN treatment (Fig. 2C). In addition, we did not observe a significant increase in the number of immune cells e.g. macrophages and lymphocytes in the hearts of the HFD/STZ group compared with that in the normal control group, which might indicate that direct inflammation might be involved in the cardiomyocytes of the HFD/STZ mice.

The serum BNP, CK, and LDH levels are three key blood biochemical indexes used in the clinical diagnosis of HF, that indicates abnormal left ventricular volume and myocardial injury (21). We analyzed the heart morphological features using pathological staining and also detected the blood biochemical indexes (i.e., $\mathrm{BNP}, \mathrm{CK}$, and $\mathrm{LDH}$ ) to confirm the effects of CAN against HF observed during clinical diagnosis. The change in the serum BNP level was moderately increased in the HFD/STZ control group compared with that in the normal control group; however, this increase was attenuated by CAN (Fig. 2d). The concentration of serum CK and LDH levels in the HFD/STZ group were moderately increased compared with that in the normal control group. However, CAN significantly attenuated the increased CK and LDH levels in the HFD/STZ diabetic control group after 12 weeks of treatment (Fig. 2e and f).

Inflammation plays an important role in diabetic HF development $(22,23)$. The increased TNF-a, IL-1 and IL-6 level is an important pathological characteristic in patients with $\operatorname{HF}(24,25)$. We extracted the total mRNA from the cardiac tissues and detected the mRNA expression levels of the inflammatory cytokines, II-1a, II-6, and Tnf- $a$ in the HFD/STZ-induced diabetic mice. The results showed that these inflammatory mRNAs in the heart tissue of the HFD/STZ diabetic group were increased by 2-3-fold (Fig. 2g-i) compared with that in the normal control group. However, this increase was significantly decreased after CAN treatment.

\subsection{The protective effect of CAN on PA-induced HL-1 cell oxidative stress, inflammation and apoptosis in vitro}

PA is a saturated fatty acid, which could induce cardiomyocyte inflammation, oxidative stress, and apoptosis in vitro (26). To investigate cardiomyocyte oxidative stress, inflammation and lipotoxicity in vivo, induced by a HFD, we used the PA-treated myocardial cell line, HL-1 and evaluated the protective effects of CAN on PA-induced cell hypertrophy, cell viability, oxidative stress and inflammatory damage. PA significantly increased cell hypertrophy induced by PA in vitro and CAN significantly inhibited cell hypertrophy (Fig. $3 a$ and $\mathrm{C}$ ). ROS was used to evaluate oxidative stress and also apoptosis. PA significantly promoted the production of ROS in the HL-1 cells; however, CAN significantly attenuated the generation of ROS (Fig. 3b). MTT assays indicated that cell viability was significantly lower in cells treated with PA compared with that in the normal control group, but that CAN significantly attenuated this effect (Fig. 3d). LDH accumulation is an important pathological characteristic in cardiomyocyte damage. Therefore, we detected LDH activity from the HL-1 cell medium in the different experimental groups. The results showed that the LDH activity in the PA-treated cells was higher compared with that in the normal control group, but CAN significantly decreased LDH accumulation in PA-treated cells (Fig. 3e). 
Furthermore, flow cytometry analysis showed that CAN could inhibit necrosis and apoptosis induced by PA (Fig. 3I and J). These results indicated that PA lowered cell viability caused by lipotoxicity, but that CAN exerted protective effects. Following which, we treated the HL-1 cells with for $24 \mathrm{~h}$ and then detected the mRNA expression levels of inflammatory cytokines. RT-qPCR assays found that PA significantly induced the mRNA expression levels of $I I-1 a, \|-6$, and Tnf-a compared with that in the normal control groups; however, this increase was significantly inhibited by CAN, in a dose-dependent manner (Fig. 3f-h). These results were consistent with the results in vivo. Notably, we found CAN could inhibit HL-1 cell viability, oxidative stress and inflammatory damage; however, we did not determine that CAN could significantly reduce lipid accumulation in the HL-1 cells induced by PA (data not shown). These results indicated that CAN directly inhibited cardiomyocyte cell lipotoxicity in vitro and in vivo; however, this was not completely dependent on the regulation of lipid metabolism.

\subsection{CAN exerted protective effects through HIF-1 signaling pathway and inhibited mTOR phosphorylation} in vivo and in vitro.

CAN is a selective SGLT2 inhibitor, and we proved its significant protective effects on lipotoxicity in cardiocytes induced by a HFD or a fatty acid in vivo and in vitro, respectively, in this research. These results might explain the beneficial effects of CAN in diabetic HF patients in clinical research. However, the potential molecular mechanisms remained unclear. SwissTargetPrediction is a web server to predict the drug target for small molecules, which was used in this study and the results revealed 102 possible targets of CAN. Next, we analyzed the 102 possible targets using ClueGo and the top 20 enriched signaling pathways are shown in Fig. 4a. There were 13,747 diabetes-related genes and 12,522 HF-related genes identified using GeneCards and the enriched pathways of top 200 genes were subsequently analyzed using ClueGo. The top 20 diabetes- and HF-related pathways are shown in Fig. $4 \mathrm{~b}$ and $4 \mathrm{c}$, respectively. The common or overlapping pathways identified in Fig. 4a-c were analyzed using the Venn Graph Application in Origin Pro 2018c (Fig. 4d). We found that the HIF-1 signaling pathway was a common signaling pathway and might be the potential target pathway of CAN in clinical diabetic HF treatment (Fig. 4d).

Based on the aforementioned bioinformatics analysis, we selected the HIF-1 signaling pathway for the preliminary investigation. HIF-1a is a key target and plays an important role in oxidative stress, inflammation, and cardiovascular disease (27). We first detected the HIF-1 a mRNA and protein expression levels in vivo and in vitro to verify the prediction. The results showed that higher levels of HIF-1a protein and mRNA expression were observed in the hearts of the HFD/STZ-induced diabetic mice compared with that in the normal control group, but CAN could inhibit this effect in vivo (Fig. 4e, $\mathrm{f}$ and k). PA increased HIF-1a mRNA and protein expression level compared with that in the blank control group; however, CAN significantly reduced the HIF-1 a mRNA and protein expression levels, in a dose-dependent manner compared with that in the PA-treated group (Fig. 4h, i, and I) in vitro. As the HIF-1 a mRNA expression level was changed significantly, CAN might inhibit HIF-1a expression by affecting an upstream target of HIF-1a in the HIF-1 signaling pathway. mTOR is an important mediator in cell metabolism, autophagy and inflammation. mTOR phosphorylation and activation was found to regulate HIF-1a transcription in the 
HIF-1 signaling pathway (11). Therefore, we next detected mTOR and p-mTOR (Ser 2448) protein expression levels in the HL-1 cell line and in the heart tissue from the HFD/STZ-induced diabetic mouse model. The in vivo results proved our hypothesis that CAN could inhibit the increase in mTOR phosphorylation in the HFD/STZ-induced mouse hearts (Fig. $4 \mathrm{~g}$ and $\mathrm{k}$ ) and in PA-treated HL-1 cell (Fig. 4j and I). We further confirmed that CAN inhibited mTOR phosphorylation using the immunofluorescence assay; CAN inhibited mTOR phosphorylation and further inhibited HIF-1a protein expression level in PAtreated HL-1 cell (Fig. 4m). We have proven that CAN could inhibit HIF-1a expression level by inhibiting mTOR phosphorylation. However, whether mTOR was the potential target of CAN in the HIF-1 signaling pathway requires further investigation.

\subsection{CAN might inhibit mTOR phosphorylation by binding to mTOR directly in the HIF-1 signaling pathway.}

Next, we investigated the potential interaction between CAN and mTOR using affinity chromatography and molecular docking. RAPA is a mTOR inhibitor and inhibits mTOR phosphorylation by binding to the FKBP-RAPA-binding (FRB) domain within mTOR (28). We compared the affinity and binding site of CAN and RAPA to the FRB domain of mTOR using SwissDock. The results showed that CAN and RAPA could bind to the same region of the mTOR FRB domain (Fig. 5c-e); a hydrophobic pocket, which is comprised of GLN49, TRP9, ILE7 and Arg4 amino acids, with similar predicted affinity (Gibbs free energy) (Fig. 5f). Subsequently, we verified the interaction between CAN and mTOR using affinity chromatography and western blot analysis. The results showed that CAN and mTOR might have a physical interaction with each other (Fig. 5a and b). These results indicated that CAN might inhibit mTOR phosphorylation by binding to the mTOR FRB domain, which is the same as with RAPA. Due to the low expression level of the SGLT2 receptor in the hearts of humans and mice (29), mTOR might be a new target of CAN in cardiovascular diseases, independent of the SGLT2 receptor.

\section{6 mTOR and HIF-1a inhibitors are a potential strategy in heart lipotoxicity protection}

Chronic activation of the mTOR and HIF-1 signaling pathway might mediate lipotoxicity in diabetic cardiocytes. LW6 is a specific HIF-1 inhibitor, which promotes the proteasomal degradation of wild-type HIF-1a by affecting HIF-1a protein stability (30). RAPA could inhibit mTOR phosphorylation and further inhibit the downstream HIF-1 a transcription and protein expression level (31-33). Next, we detected the cell survival protection and anti-inflammation effects of the HIF-1 $a$ and mTOR inhibitors in the PAinduced HL-1 cell line to investigate the effects of HIF-1a on PA-induced inflammation and cellular toxicities. The results showed that RAPA and LW6 prevented cell death induced by PA (Fig. 6a and f) and significantly reduced the mRNA expression level of $I I-1 a, I I-6$, and Tnf- $a$ (Fig. 6b-d and g-i). LW6 did not significantly change the HIF-1 a mRNA expression level (Fig. 6e), but inhibited the HIF-1 a protein function (Fig. 6k and I) as previously reported (30). Similar to CAN, RAPA could significantly inhibit the increase of HIF-1 a mRNA and protein expression levels induced by PA (Fig. 6j-I). A previous study also found that RAPA exerted cardioprotective effects on myocardial dysfunction during sepsis induced by cecal ligation and puncture in rats through the mTOR-HIF-1 $\mathrm{a}$ axis and autophagy (34). However, increasing research has found an association between high HIF-a expression level and inflammation, metabolic and 
cardiovascular disease, and the HIF-1a inhibitor was considered to be a potential therapeutic strategy (35). These results indicated that inhibition of the mTOR or HIF-1 a signaling pathway could prevent lipotoxicity in the heart and serve as an important target. CAN might have similar pharmacological activities, similar to these mTOR inhibitors.

\section{Discussion}

Diabetic cardiomyopathy was found to increase fat accumulation and oxidative metabolism, which causes an increased production of mitochondrial ROS $(36,37)$. Cardiomyocyte lipotoxicity is an important risk factor in diabetic cardiomyopathy development (38). Many dietary saturated fatty acids, such as PA, exist in numerous types of unhealthy food, and induce lipid accumulation and lipotoxicity in the heart (39). Therefore, in this study, we used HFD, combined with STZ, to induce the animal model of diabetic cardiomyopathy in mice. Impaired glucose metabolism also lead to lipid accumulation and FAO increase in diabetic hearts (40), and the increased FAO further promoted mitochondrial ROS generation. CAN inhibited energy metabolism by targeting mitochondrial complex 1 (41), in an off-target manner, which was independent of the SGLT2 receptor. In our previous studies, CAN significantly inhibited intracellular glucose metabolism $(13,42)$. These phenomena might partly explain why CAN inhibited ROS production, as observed in this study.

Impaired glucose metabolism, increased lipid accumulation and ROS levels might cause hypoxia and chronic pathological expression level of HIF-1a in the heart (43) (44). Chronic activation of HIF-1a was responsible for recruiting $\mathrm{M} 1$ macrophages in the heart and mediated metabolic inflammation in cardiomyocytes, thereby releasing IL-6, MCP-1, TNF- $a$, and IL-1 $\beta$, NADPH oxidase, and connective tissue growth factor; the resulting chronic inflammation accelerated cardiac fibrosis and impaired the cardiac diastolic function (43) (45). Recently, research found that knockout of HIF-1a protected HF in the animal model (9). As identified by the bioinformatics analysis, the HIF-1 a signaling pathway could play an important role in the pathogenesis of diabetes and HF. The reduction in ROS generation by CAN might promote HIF-1a degradation, decrease the stability of HIF-1a, and inhibit the expression level of inflammatory factors induced by lipotoxicity.

mTOR serves as a central regulator of lipid storage and metabolism (46). High-fat uptake promoted mTOR phosphorylation (47). Following mTOR activation, the accumulation of triglycerides is facilitated by the increase in adipogenesis and lipogenesis (48), which also contributed to lipotoxicity. The inhibition of mTOR has been hypothesized to have beneficial effects against atherosclerosis, cardiac hypertrophy and HF (10). Furthermore, mTOR could directly activate HIF-1 a transcription and upregulate the HIF-1a protein expression level, and conversely, the inhibition of mTOR by RAPA attenuated the HIF-1a expression level (49). Therefore, a promising strategy in the treatment of HF induced by lipotoxicity is to target the mTOR/HIF-1a axis.

In this study, we proved that RAPA (mTOR inhibitor) and LW6 (HIF-1a inhibitor) exerted significant antiinflammatory activities in the PA-induced HL-I cell line. This result indicated that the mTOR/HIF-1a 
mediator could alleviate the development of cardiomyocyte inflammation and cell toxicities induced by lipid overload. Compared with that in the mTOR and HIF-1a inhibitors, CAN could inhibit mTOR phosphorylation and HIF-1a transcription and expression, similar to RAPA. Furthermore, we used molecular docking and affinity chromatography methods to confirm that CAN had a direct molecular interaction with the FRB domain of mTOR and might be a new inhibitor of mTOR phosphorylation.

This study has some limitations. Firstly, we used young male C57BI/6 mice as these young animals were sensitive to HFD and STZ and were easy to maintain. However, the majority of patients receiving SGLT2 inhibitors will be older, not young and healthy; therefore, it is difficult to extrapolate these findings to patients with heart disease. Secondly, $\mathrm{C} 57 \mathrm{BI} / 6$ mice may be resistant to diabetic complications and require a long time to induce complications. In particular, with this model, we did not observe a significant increase in the immune cells in the heart tissues; however, the protein and mRNA expression level of inflammatory factors were increased in these cardiomyocytes. A more suitable animal model is required in the future. Thirdly, the exact molecular docking mechanism e.g. the binding sites between CAN and the amino acids of the mTOR protein requires further identification in the future.

\section{Conclusion}

In summary, we confirmed the protective effects of CAN on lipotoxicity, e.g. oxidative stress, inflammation, and cellular toxicities in the hearts of the diabetic C57BL/ 6 mouse models induced by HFD/STZ and in the PA-treated HL-1 cell line. The molecular mechanisms of CAN might be mediated by directly binding to the mTOR protein and inhibiting mTOR phosphorylation, then inhibiting HIF-1a mRNA and protein expression levels. This research indicated that the mTOR/HIF-1a signaling pathway could be involved in lipotoxicity in the hearts of diabetic mice. Furthermore, CAN exerted protective effects on lipotoxicity in the hearts of diabetic mice, which was partly mediated by regulating the mTOR/HIF-1a signaling pathway. These results may partly explain why CAN significantly reduced the number of HF events in diabetic patients and provide a promising target in the development of a new drug against diabetic HF. The SGLT2 inhibitor might have a new drug target by targeting the mTOR/HIF-1a pathway in the cardiovascular system, which is independent of the kidney SGLT2 receptor.

\section{Abbreviations}

CAN, canagliflozin

RAPA, rapamycin

PA, palmitic acid

STZ, streptozotocin

$H F$, heart failure 
T2DM, type 2 diabetes mellitus

HFD, high-fat diet

LDH, lactate dehydrogenase

CK, creatine kinase

ROS, reactive oxygen species

BNP, B-type natriuretic peptide

\section{Declarations}

\section{(1) Ethics approval and consent to participate}

The study was performed in strict accordance with the National Institutes of Health Guide for the Care and Use of Laboratory Animals and the protocol was approved by the Bioethics Committee of Shenzhen International Graduate School, Tsinghua University, China (Ethics issue [2020] No. 9).

\section{(2) Consent for publication}

Not applicable.

\section{(3) Availability of data and materials}

The datasets used and/or analyzed during the current study are available from the corresponding author on reasonable request.

\section{(4) Competing interests}

The authors declare that they have no competing interests.

\section{(5) Funding}

This work was supported by the National Natural Science Foundation of China (No. 91957110; 81373460), Shenzhen Science and Technology Innovation Committee (JSGG20200519160752002; JCYJ20170307152357168), and the Natural Science Foundation of Guangdong Province (No. 2014A030313744).

\section{(6) Author Contributions}

Pengbo Sun contributed investigation, data curation, data analysis and manuscript writing; Yipei Ding contributed investigation and data validation; Jingyi Luo contributed Investigation; Jin Zhong contributed investigation; Weidong Xie contributed project administration, supervision, conceptualization, data analysis, manuscript writing and revision. 


\section{(7) Acknowledgements}

Not applicable

\section{References}

1. Heintjes EM, Houben E, Beekman-Hendriks WL, Lighaam E, Cremers SM, Penning-van Beest FJA, et al. Trends in mortality, cardiovascular complications, and risk factors in type 2 diabetes. Neth J Med. 2019;77(9):317-29.

2. Bugger $\mathrm{H}$, Abel ED. Molecular mechanisms of diabetic cardiomyopathy. Diabetologia. 2014;57(4):660-71.

3. Lopaschuk GD, Belke DD, Gamble J, Itoi T, Schonekess BO. Regulation of fatty acid oxidation in the mammalian heart in health and disease. Biochim Biophys Acta. 1994;1213(3):263-76.

4. Fillmore N, Mori J, Lopaschuk GD. Mitochondrial fatty acid oxidation alterations in heart failure, ischaemic heart disease and diabetic cardiomyopathy. Br J Pharmacol. 2014;171(8):2080-90.

5. Rijzewijk LJ, van der Meer RW, Smit JW, Diamant M, Bax JJ, Hammer S, et al. Myocardial steatosis is an independent predictor of diastolic dysfunction in type 2 diabetes mellitus. J Am Coll Cardiol. 2008;52(22):1793-9.

6. Rådholm K, Figtree G, Perkovic V, Solomon SD, Mahaffey KW, de Zeeuw D, et al. Canagliflozin and Heart Failure in Type 2 Diabetes Mellitus: Results From the CANVAS Program. Circulation. 2018;138(5):458-68.

7. Lim VG, Bell RM, Arjun S, Kolatsi-Joannou M, Long DA, Yellon DM. SGLT2 Inhibitor, Canagliflozin, Attenuates Myocardial Infarction in the Diabetic and Nondiabetic Heart. JACC Basic Transl Sci. 2019;4(1):15-26.

8. Inoue M, Hayashi A, Taguchi T, Arai R, Sasaki S, Takano K, et al. Effects of canagliflozin on body composition and hepatic fat content in type 2 diabetes patients with non-alcoholic fatty liver disease. J Diabetes Investig. 2019;10(4):1004-11.

9. Kumar S, Wang G, Liu W, Ding W, Dong M, Zheng N, et al. Hypoxia-Induced Mitogenic Factor Promotes Cardiac Hypertrophy via Calcium-Dependent and Hypoxia-Inducible Factor-1 alpha Mechanisms. Hypertension. 2018;72(2):331-42.

10. Sanches-Silva A, Testai L, Nabavi SF, Battino M, Pandima Devi K, Tejada S, et al. Therapeutic potential of polyphenols in cardiovascular diseases: Regulation of mTOR signaling pathway. Pharmacol Res. 2020;152:104626.

11. Land SC, Tee AR. Hypoxia-inducible factor 1 alpha is regulated by the mammalian target of rapamycin (mTOR) via an mTOR signaling motif. J Biol Chem. 2007;282(28):20534-43.

12. Xie W, Nie Y, Du L, Zhang Y, Cai G. Preventive effects of fenofibrate on insulin resistance, hyperglycaemia, visceral fat accumulation in $\mathrm{NIH}$ mice induced by small-dose streptozotocin and lard. Pharmacol Res. 2007;55(5):392-9. 
13. Xu C, Wang W, Zhong J, Lei F, Xu N, Zhang Y, et al. Canagliflozin exerts anti-inflammatory effects by inhibiting intracellular glucose metabolism and promoting autophagy in immune cells. Biochem Pharmacol. 2018;152:45-59.

14. Cui K, Zhang S, Jiang X, Xie W. Novel synergic antidiabetic effects of Astragalus polysaccharides combined with Crataegus flavonoids via improvement of islet function and liver metabolism. Mol Med Rep. 2016;13(6):4737-44.

15. Daina A, Michielin O, Zoete V. SwissTargetPrediction: updated data and new features for efficient prediction of protein targets of small molecules. Nucleic Acids Res. 2019;47(W1):W357-W64.

16. Bindea G, Mlecnik B, Hackl H, Charoentong P, Tosolini M, Kirilovsky A, et al. ClueGO: a Cytoscape plug-in to decipher functionally grouped gene ontology and pathway annotation networks. Bioinformatics. 2009;25(8):1091-3.

17. Grosdidier A, Zoete V, Michielin O. SwissDock, a protein-small molecule docking web service based on EADock DSS. Nucleic Acids Res. 2011;39(Web Server issue):W270-7.

18. Chen F, Zhu K, Chen L, Ouyang L, Chen C, Gu L, et al. Protein target identification of ginsenosides in skeletal muscle tissues: discovery of natural small-molecule activators of muscle-type creatine kinase. J Ginseng Res. 2020;44(3):461-74.

19. Liu Q, Wang S, Cai L. Diabetic cardiomyopathy and its mechanisms: Role of oxidative stress and damage. J Diabetes Investig. 2014;5(6):623-34.

20. O'Grady H, Mostafa K, Zafar H, Lohan D, Morris L, Sharif F. Changes in left ventricular shape and morphology in the presence of heart failure: a four-dimensional quantitative and qualitative analysis. Int J Comput Assist Radiol Surg. 2019;14(8):1415-30.

21. Nalban N, Sangaraju R, Alavala S, Mir SM, Jerald MK, Sistla R. Arbutin Attenuates IsoproterenolInduced Cardiac Hypertrophy by Inhibiting TLR-4/NF-KB Pathway in Mice. Cardiovasc Toxicol. 2020;20(3):235-48.

22. Rana JS, Nieuwdorp M, Jukema JW, Kastelein JJ. Cardiovascular metabolic syndrome - an interplay of, obesity, inflammation, diabetes and coronary heart disease. Diabetes Obes Metab. 2007;9(3):21832.

23. Aimo A, Castiglione V, Borrelli C, Saccaro LF, Franzini M, Masi S, et al. Oxidative stress and inflammation in the evolution of heart failure: From pathophysiology to therapeutic strategies. Eur $\mathrm{J}$ Prev Cardiol. 2020;27(5):494-510.

24. Zhang Y, Bauersachs J, Langer HF. Immune mechanisms in heart failure. Eur J Heart Fail. 2017;19(11):1379-89.

25. Jones DP, Patel J. Therapeutic Approaches Targeting Inflammation in Cardiovascular Disorders. Biology (Basel). 2018;7(4).

26. Mangali S, Bhat A, Udumula MP, Dhar I, Sriram D, Dhar A. Inhibition of protein kinase R protects against palmitic acid-induced inflammation, oxidative stress, and apoptosis through the JNK/NFkB/NLRP3 pathway in cultured H9C2 cardiomyocytes. J Cell Biochem. 2019;120(3):3651-63. 
27. Abe H, Semba H, Takeda N. The Roles of Hypoxia Signaling in the Pathogenesis of Cardiovascular Diseases. J Atheroscler Thromb. 2017;24(9):884-94.

28. Marz AM, Fabian AK, Kozany C, Bracher A, Hausch F. Large FK506-binding proteins shape the pharmacology of rapamycin. Mol Cell Biol. 2013;33(7):1357-67.

29. Vrhovac I, Balen Eror D, Klessen D, Burger C, Breljak D, Kraus O, et al. Localizations of Na(+)-D-glucose cotransporters SGLT1 and SGLT2 in human kidney and of SGLT1 in human small intestine, liver, lung, and heart. Pflugers Arch. 2015;467(9):1881-98.

30. Lee K, Kang JE, Park SK, Jin Y, Chung KS, Kim HM, et al. LW6, a novel HIF-1 inhibitor, promotes proteasomal degradation of HIF-1alpha via upregulation of VHL in a colon cancer cell line. Biochem Pharmacol. 2010;80(7):982-9.

31. Dodd KM, Yang J, Shen MH, Sampson JR, Tee AR. mTORC1 drives HIF-1 alpha and VEGF-A signalling via multiple mechanisms involving 4E-BP1, S6K1 and STAT3. Oncogene. 2015;34(17):2239-50.

32. Nakazawa H, Ikeda K, Shinozaki S, Kobayashi M, Ikegami Y, Fu M, et al. Burn-induced muscle metabolic derangements and mitochondrial dysfunction are associated with activation of HIF1alpha and mTORC1: Role of protein farnesylation. Sci Rep. 2017;7(1):6618.

33. Mi C, Ma J, Shi H, Li J, Wang F, Lee JJ, et al. 4',6-dihydroxy-4-methoxyisoaurone inhibits the HIF1alpha pathway through inhibition of Akt/mTOR/p70S6K/4E-BP1 phosphorylation. J Pharmacol Sci. 2014;125(2):193-201.

34. Han W, Wang H, Su L, Long Y, Cui N, Liu D. Inhibition of the mTOR Pathway Exerts Cardioprotective Effects Partly through Autophagy in CLP Rats. Mediators Inflamm. 2018;2018:4798209.

35. Majmundar AJ, Wong WJ, Simon MC. Hypoxia-inducible factors and the response to hypoxic stress. Mol Cell. 2010;40(2):294-309.

36. Kaludercic N, Di Lisa F. Mitochondrial ROS Formation in the Pathogenesis of Diabetic Cardiomyopathy. Front Cardiovasc Med. 2020;7:12.

37. Palomer X, Salvadó L, Barroso E, Vázquez-Carrera M. An overview of the crosstalk between inflammatory processes and metabolic dysregulation during diabetic cardiomyopathy. Int $\mathrm{J}$ Cardiol. 2013;168(4):3160-72.

38. Tong M, Saito T, Zhai P, Oka SI, Mizushima W, Nakamura M, et al. Mitophagy Is Essential for Maintaining Cardiac Function During High Fat Diet-Induced Diabetic Cardiomyopathy. Circ Res. 2019;124(9):1360-71.

39. Dalla Valle A, Vertongen P, Spruyt D, Lechanteur J, Suain V, Gaspard N, et al. Induction of StearoylCoA 9-Desaturase 1 Protects Human Mesenchymal Stromal Cells Against Palmitic Acid-Induced Lipotoxicity and Inflammation. Front Endocrinol (Lausanne). 2019;10:726.

40. Goldberg IJ, Trent CM, Schulze PC. Lipid metabolism and toxicity in the heart. Cell Metab. 2012;15(6):805-12.

41. Secker PF, Beneke S, Schlichenmaier N, Delp J, Gutbier S, Leist M, et al. Canagliflozin mediated dual inhibition of mitochondrial glutamate dehydrogenase and complex I: an off-target adverse effect. Cell Death Dis. 2018;9(2):226. 
42. Zhong J, Sun P, Xu N, Liao M, Xu C, Ding Y, et al. Canagliflozin inhibits p-gp function and early autophagy and improves the sensitivity to the antitumor effect of doxorubicin. Biochem Pharmacol. 2020;175:113856.

43. Warbrick I, Rabkin SW. Hypoxia-inducible factor 1-alpha (HIF-1 alpha) as a factor mediating the relationship between obesity and heart failure with preserved ejection fraction. Obes Rev. 2019;20(5):701-12.

44. Movafagh S, Crook S, Vo K. Regulation of hypoxia-inducible factor-1 a by reactive oxygen species: new developments in an old debate. J Cell Biochem. 2015;116(5):696-703.

45. Warbrick I, Rabkin SW. Hypoxia-inducible factor 1-alpha (HIF-1a) as a factor mediating the relationship between obesity and heart failure with preserved ejection fraction. Obes Rev. 2019;20(5):701-12.

46. Chakrabarti P, Kandror KV. The role of mTOR in lipid homeostasis and diabetes progression. Curr Opin Endocrinol Diabetes Obes. 2015;22(5):340-6.

47. Kling DN, DeBose-Scarlett EM, Teixeira LD, Gezan SA, Lorca GL, Gonzalez CF. Sex Modulates Lactobacillus johnsonii N6.2 and Phytophenol Effectiveness in Reducing High Fat Diet Induced mTOR Activation in Sprague-Dawley Rats. Front Microbiol. 2018;9:2649.

48. Caron A, Richard D, Laplante M. The Roles of mTOR Complexes in Lipid Metabolism. Annu Rev Nutr. 2015;35:321-48.

49. Chen H, Xiong T, Qu Y, Zhao F, Ferriero D, Mu D. mTOR activates hypoxia-inducible factor-1 $a$ and inhibits neuronal apoptosis in the developing rat brain during the early phase after hypoxia-ischemia. Neurosci Lett. 2012;507(2):118-23.

\section{Tables}

Table 1. Primers for mRNA q-PCR 


\begin{tabular}{|lllll|}
\hline Gene & Forward & Reverse & NCBI & Size \\
\hline Hif-1a & ACCTTCATCGGAAAC & ACTGTTAGGCTCAGG & NM_001313919.1 & 156 \\
(mouse) & TCCAAAG & TGAACT & & \\
II-1a & TCTGCCATTGACCATC & ATCTTCCCGTTGCTTG & NM_010554.4 & 182 \\
(mouse) & TC & AC & & \\
II-6 & CTGCAAGAGACTTCC & GAGTGGTATAGACAG & NM_031168 & 131 \\
(mouse) & ATCCAG & GTCTGTTGG & & \\
\hline Tnf- $a$ & GGGCTTCCAGAACTC & GCTACAGGCTTGTCAC & NM_013693.2 & 213 \\
(mouse) & CA & TCG & & \\
\hline$\beta$-actin & GTGACGTTGACATCC & GCCGGACTCATCGTAC & NM_007393 & 245 \\
(mouse) & GTAAAGA & TCC & & \\
\hline
\end{tabular}

\section{Figures}




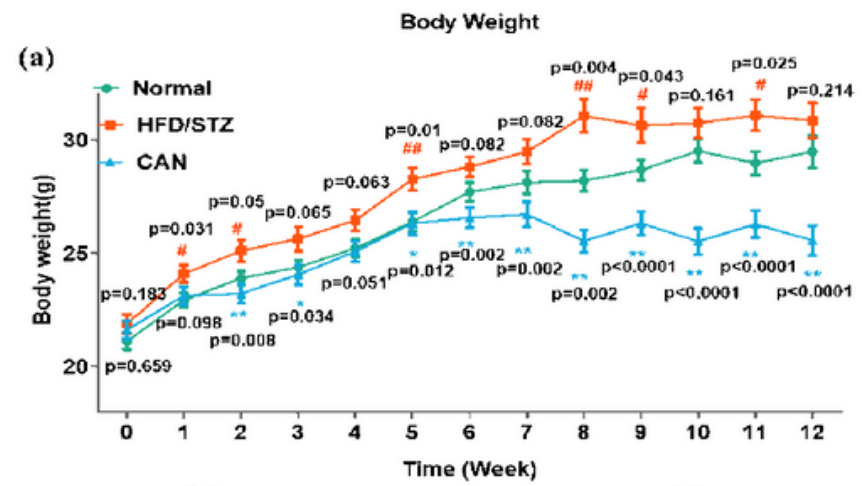

(b)

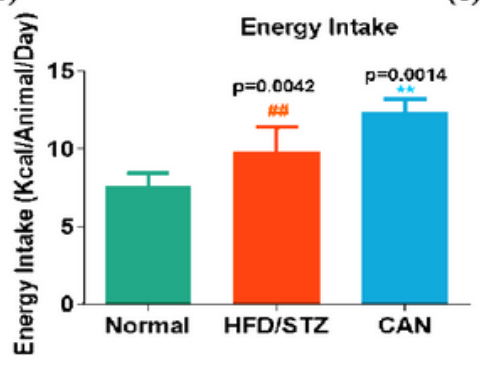

c)

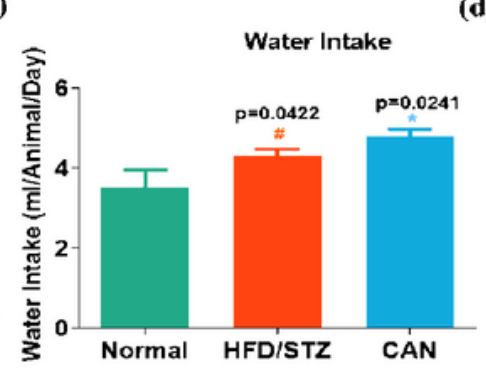

(d)

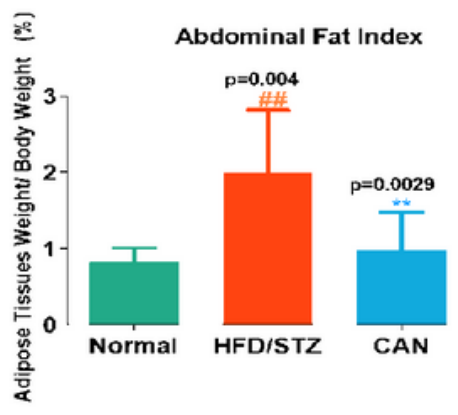

(e)

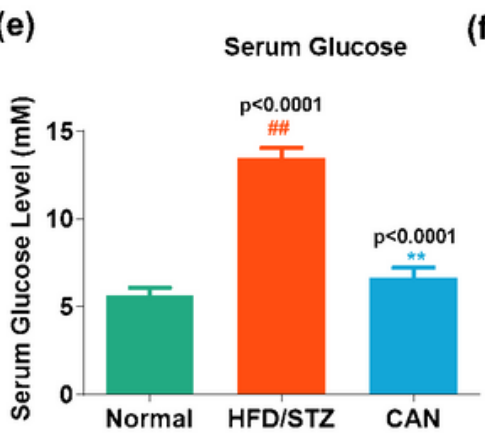

(h)

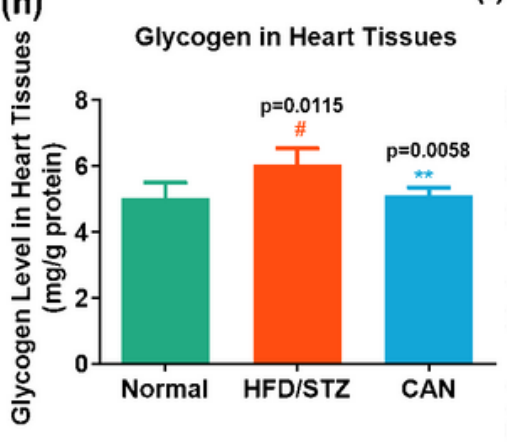

(f)

(i)
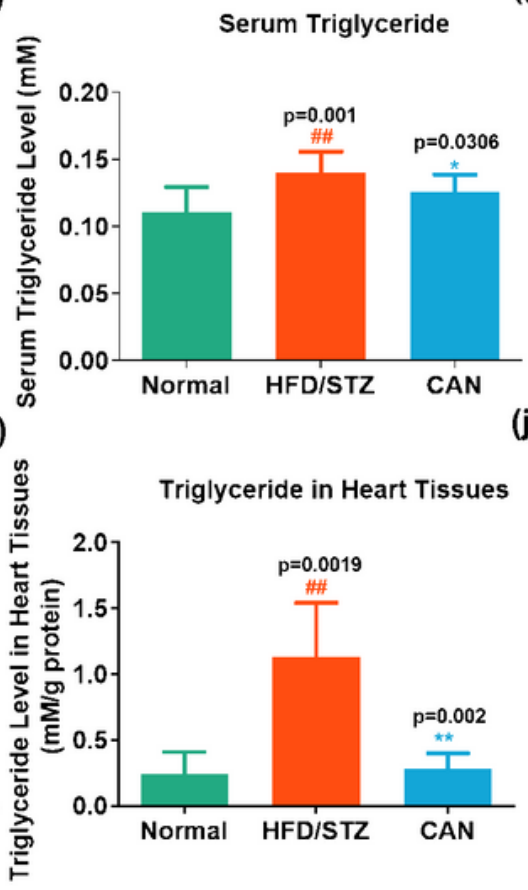

(g)

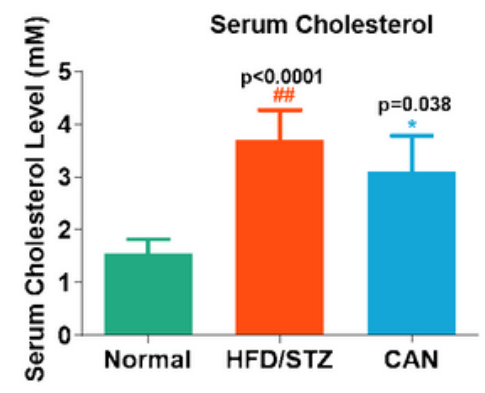

(j)

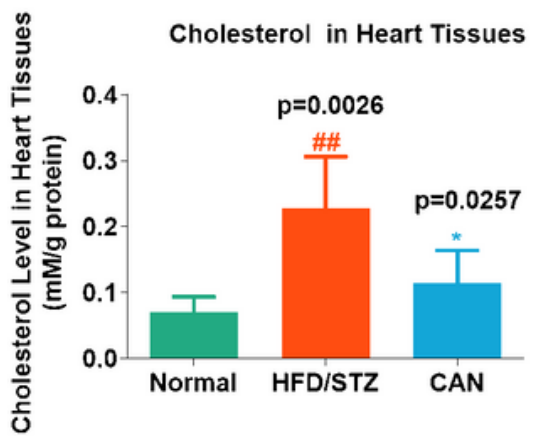

\section{Figure 1}

CAN reduced the body and adipose tissue weights, blood lipid/glucose levels and heart lipid/glucose accumulation, which was not mediated by inhibiting the dietary intake in the HFD/STZ-induced C57BL/6 mice. (a) Body weight, (b) adipose tissue weight index $(n=10)$, (c) energy intake $(n=8)$ and (d) water intake $(n=3)$, and $(e)$ serum glucose $(n=10),(f)$ serum triglyceride $(n=10),(g)$ serum cholesterol $(n=10),(h)$ glycogen ( $n=5)$, (i) triglyceride $(n=5),(j)$ cholesterol levels in the heart tissues $(n=5)$ from the HFD/STZ- 
induced diabetic mice. The data are presented as the mean $\pm S D(n=10)$. \#P<0.05 and \#\#P<0.01 vs. normal; ${ }^{*}<0.05$ and ${ }^{* *} \mathrm{P}<0.01$ vs. HFD/STZ. Normal, normal control group; HFD, high-fat diet; $S T Z$, streptozotocin; CAN, canagliflozin; HFD/STZ, HFD/STZ-induced diabetic control group; CAN, canagliflozin-treated HFD/STZ-induced diabetic group.

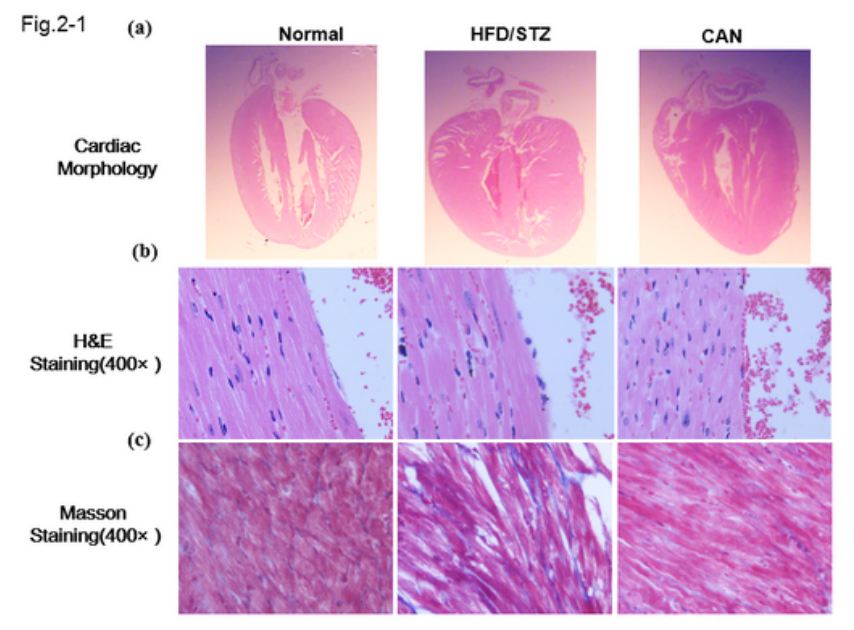

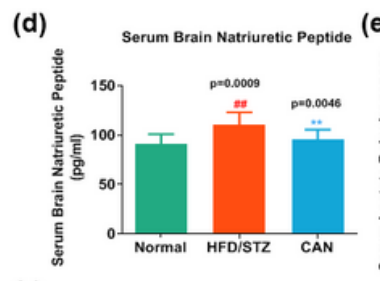

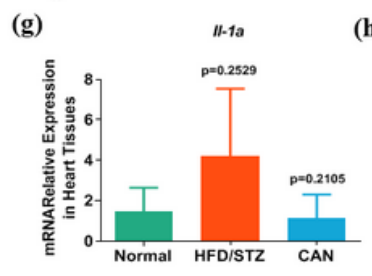

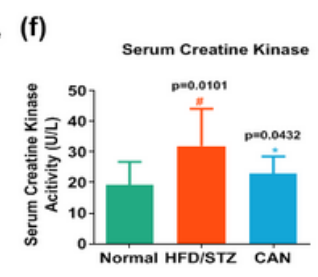

(i)
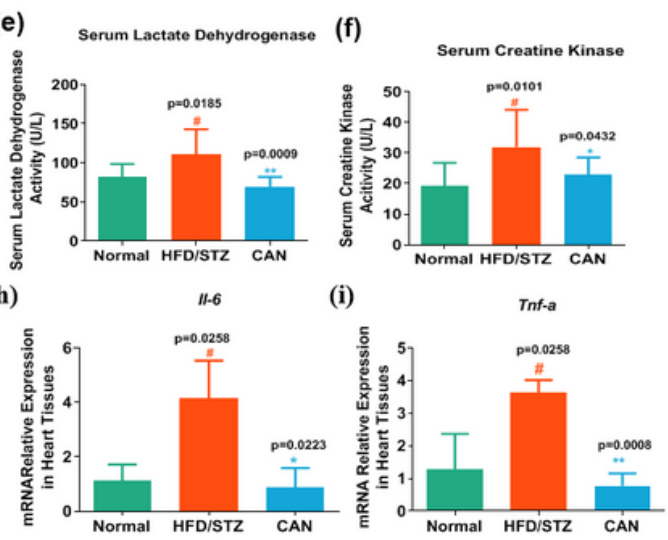

\section{Figure 2}

Protective effects of CAN in the hearts of HFD/STZ-induced diabetic mice. (a) Morphology of the hearts from the HFD/STZ-induced diabetic mice was measured using pathological slices. (b) H\&E and (c) Masson staining using pathological slices in the hearts of the HFD/STZ-induced diabetic mice. (d) BNP, (e) CK and (f) LDH levels in the serum detected by biochemical kits $(n=10)$. mRNA expression levels of the inflammatory factors, (g) II-1a, (h) II-6, and (i) Tnf-a in the hearts of the HFD/STZ-induced diabetic mice were determined using reverse transcription-quantitative PCR $(n=3)$. The data are presented as the mean $\pm S D(n=10) . \# P<0.05$ and $\# \# P<0.01$ vs. normal; ${ }^{*} P<0.05$ and ${ }^{*} * P<0.01$ vs. HFD/STZ. H\&E, hematoxylin and eosin; BNP, B-type natriuretic peptide; CK, creatine kinase; LDH, lactate dehydrogenase; HFD, high-fat diet; STZ, streptozotocin; CAN, canagliflozin; normal, normal control group. HFD/STZ, HFD/STZ-induced diabetic control group; CAN, canagliflozin-treated HFD/STZ-induced diabetic group. 


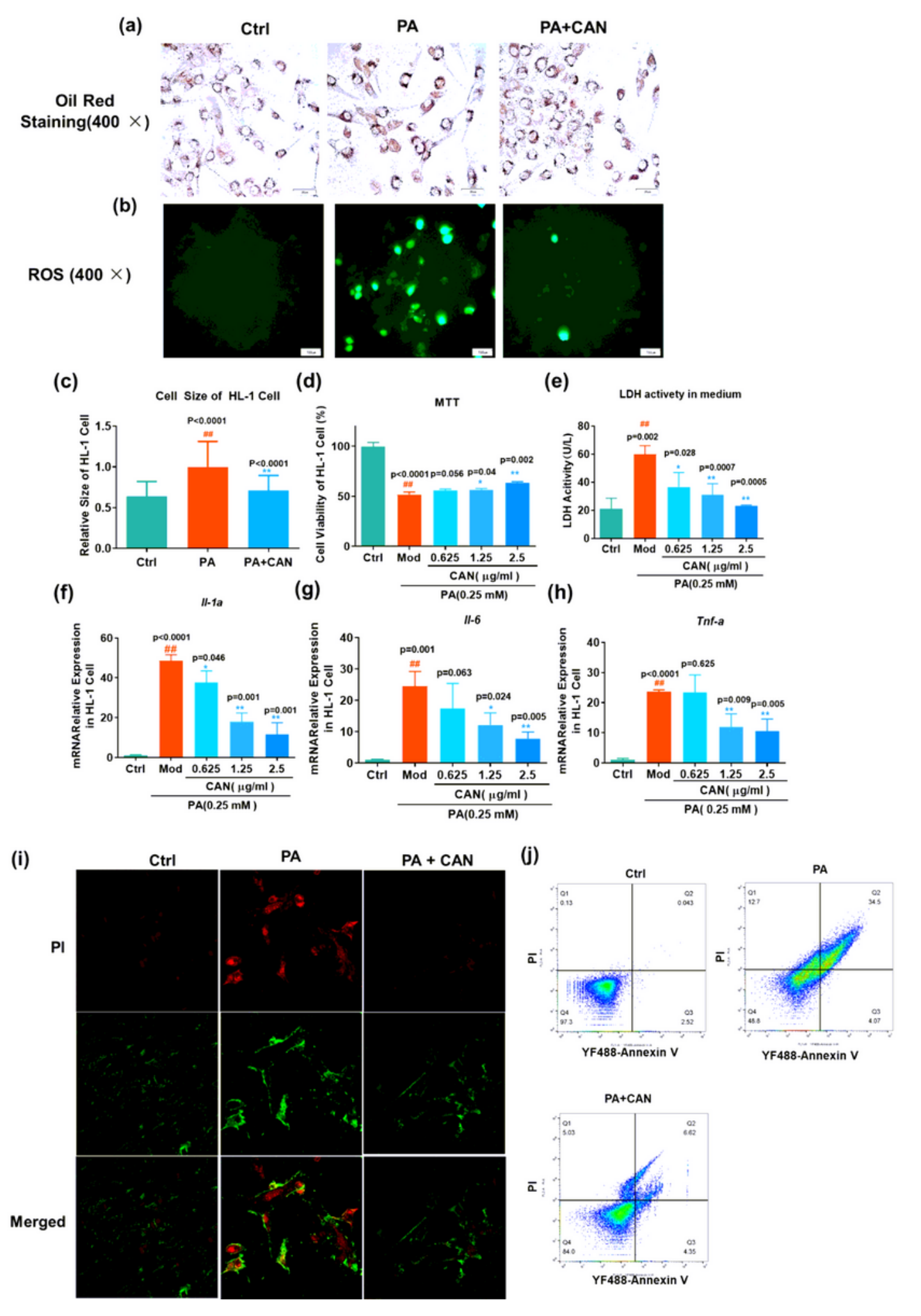

\section{Figure 3}

The protective effects of CAN on PA-induced HL-1 cells. (a) Oil red $\mathrm{O}$ staining results of the HL-1 cell line. Magnification, $x 400$. (b) Statistical results of the HL-1 cell size after different treatments. (c) ROS was detected using the fluorescent probe, 2',7'-dichlorofluorescein diacetate. Magnification, x400. (d) Cell viability in the HL-1 cells was detected using a MTT assay. (e) LDH activity in the HL-1 cell culture medium. mRNA expression levels of the inflammatory factors, (f) II-1a, (g) II-6, and (h) Tnf-a in the HL-1 
cells determined using reverse transcription-quantitative PCR. (i) Immunofluorescence staining with YF488-Annexin V and PI in the HL-1 cells, using a confocal microscope (magnification, x600). Red fluorescence indicates $\mathrm{PI}$, green fluorescence indicates YF488-annexin $\mathrm{V}$ and the merged figure indicates the merged signal of PI and YF488-annexin V. (j) Flow cytometry assay in the HL-1 cells. The data are presented as the mean $\pm S D(n=3)$. $\# P<0.05$ and \#\#P<0.01 vs. ctrl; ${ }^{*} P<0.05$ and ${ }^{*} \mathrm{P}<0.01$ vs. PA. Ctrl, untreated normal control; Mod/PA, PA-treated $(0.25 \mathrm{mM})$ control group; $0.625, \mathrm{HL}-1$ cells were treated with PA $(0.25 \mathrm{mM})$ and CAN $(0.625 \mu \mathrm{g} / \mathrm{ml}) ; 1.25, \mathrm{HL}-1$ cells were treated with PA $(0.25 \mathrm{mM})$ and CAN $(1.25$ $\mu \mathrm{g} / \mathrm{ml}) ; 2.5, \mathrm{HL}-1$ cells were treated with PA $(0.25 \mathrm{mM})$ and CAN $(2.5 \mu \mathrm{g} / \mathrm{ml})$; PA+CAN, $\mathrm{HL}-1$ cells treated with PA $(0.25 \mathrm{mM})$ and CAN $(2.5 \mu \mathrm{g} / \mathrm{ml}) ; \mathrm{ROS}$, reactive oxygen species, LDH, lactate dehydrogenase; PA, palmitic acid; CAN, canagliflozin. 

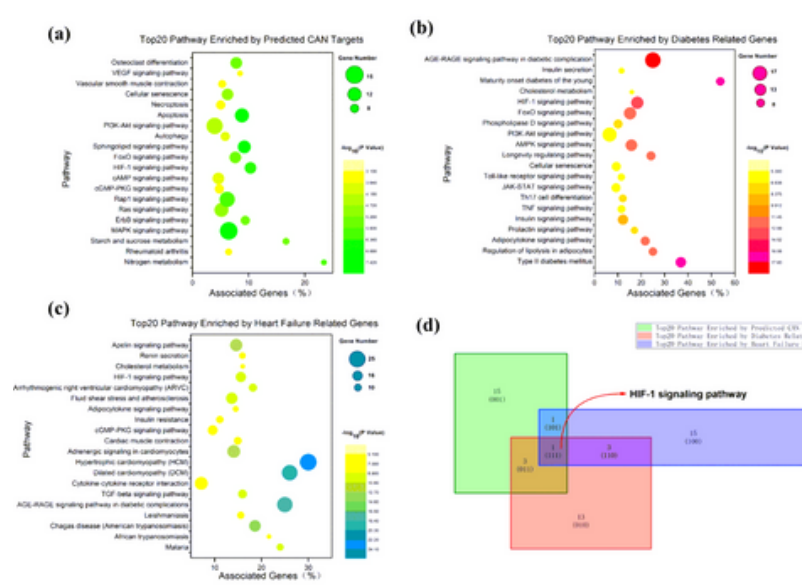

(d)
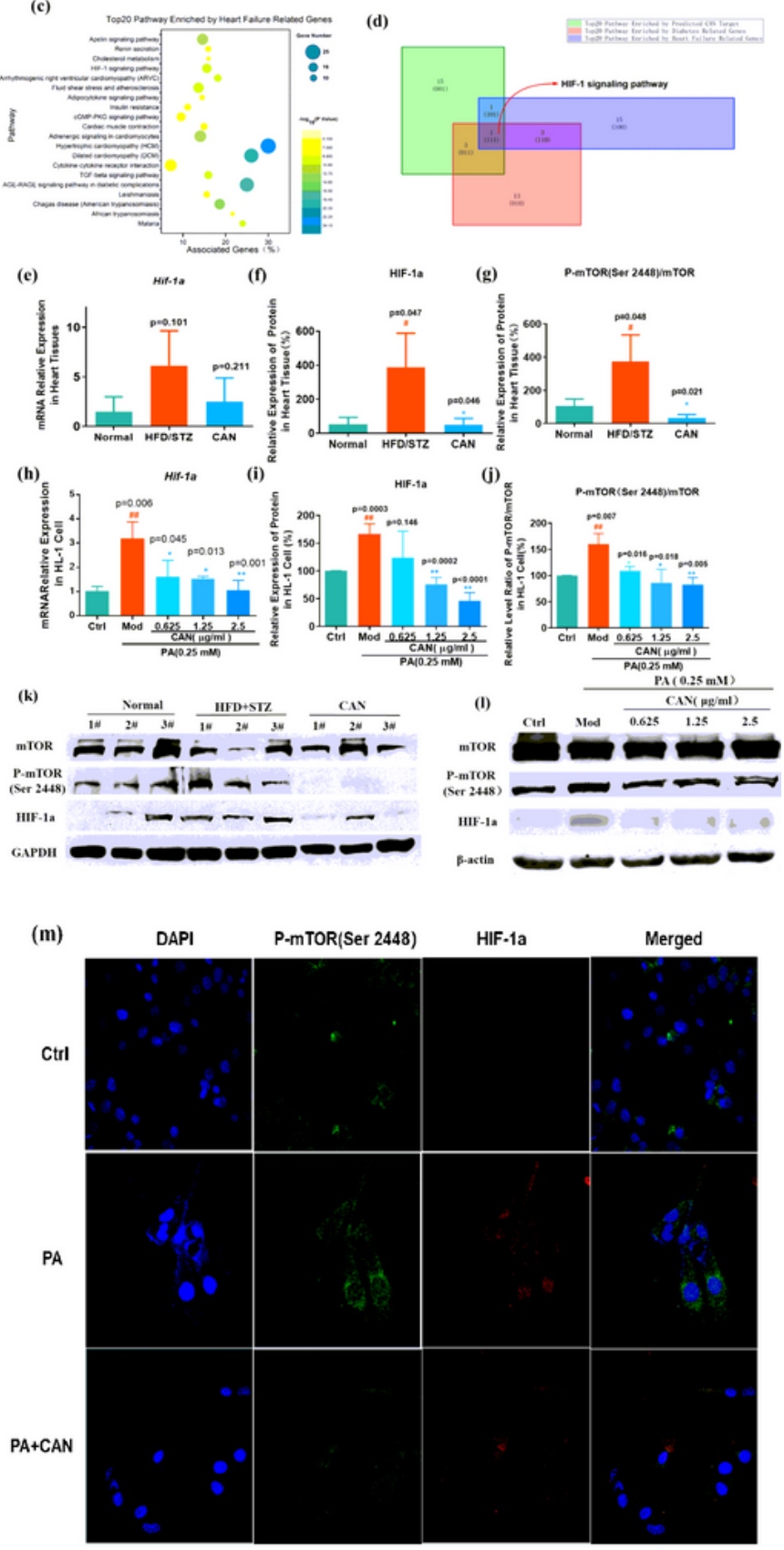

Figure 4

CAN exerted protective effects through HIF-1 signaling pathway in vivo and in vitro. (a) The top 20 enriched pathways of the predicted targets of CAN, which were identified using CluoGo. $p<0.05$. (b) The top 20 enriched pathways of the diabetes-related genes (from the top 200 using GeneCards) using CluoGo. $p<0.001$. (c) The top 20 enriched pathways of the HF-related genes (from the top 200 using GeneCards) using CluoGo, $p<0.001$. (d) The common pathways of (a), (b) and (c) were analyzed using 
Origin 2018. (e) Relative expression level of Hif-1a mRNA in the hearts of the HFD/STZ-induced diabetic mice. (f) Quantification of HIF-1a protein in the hearts of the HFD/STZ-induced diabetic mice was determined using ImageJ software following western blot analysis (k). (g) p-mTOR (Ser 2448)/mTOR protein expression ratio was determined using ImageJ following western blot analysis (k). (h) Relative expression level of Hif-1a mRNA in the HL-1 cells. (i) Quantification of HIF-1a protein in the HL-1 cells was determined using Image J software following western blot analysis (I). (j) p-mTOR (Ser 2448)/mTOR protein expression ratio in the $\mathrm{HL}-1$ cells was determined using Image $\mathrm{J}$ software following western blot analysis (I). HIF-1a, p-mTOR (Ser 2448) and mTOR expression levels in the hearts of the HFD/STZinduced diabetic mice $(\mathrm{k})$ and in the HL-1 cells (I) were determined using western blot analysis. $(\mathrm{m})$ Immunofluorescence analysis of the HL-1 cells using a confocal microscope. Magnification, $x 600$. Green fluorescence indicates p-mTOR (Ser 2448), red fluorescence indicates HIF-1a, blue fluorescence indicates DAPI and merged indicates merged signal of p-mTOR (Ser 2448), HIF-1 $a$ and DAPI. The data are presented as the mean $\pm S D$. $n=3$. $\# P<0.05$ and $\# \# P<0.01$ vs. normal control; ${ }^{*} P<0.05$ and ${ }^{*} * P<0.01$ vs. HFD/STZ or PA. Mod/PA, PA $(0.25 \mathrm{mM})$ treated control group; $0.625, \mathrm{HL}-1$ cells were treated with PA (0.25 $\mathrm{mM})$ and CAN $(0.625 \mu \mathrm{g} / \mathrm{ml}) ; 1.25, \mathrm{HL}-1$ cells were treated with PA $(0.25 \mathrm{mM})$ and CAN $(1.25 \mu \mathrm{g} / \mathrm{ml}) ; 2.5$, $\mathrm{HL}-1$ cells were treated with PA $(0.25 \mathrm{mM})$ and CAN $(2.5 \mu \mathrm{g} / \mathrm{ml})$; PA+CAN, HL- 1 cells treated with PA $(0.25$ $\mathrm{mM})$ and CAN $(2.5 \mu \mathrm{g} / \mathrm{ml}) ; \mathrm{p}$, phosphorylated.

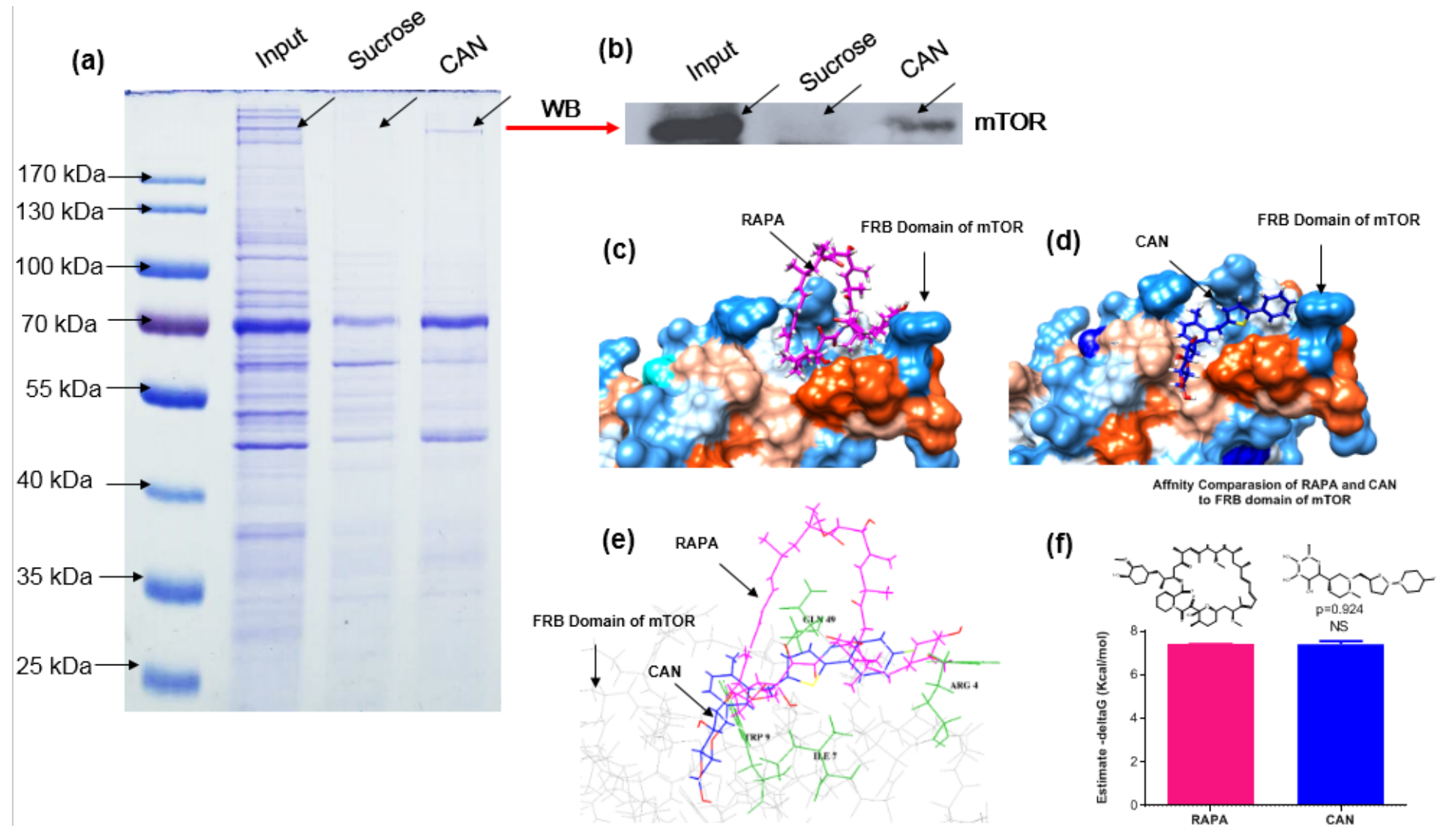

\section{Figure 5}

CAN inhibited mTOR phosphorylation by binding to the FRB domain within mTOR. (a and b) Affinity chromatography results of CAN (protein identified using western blot analysis). Molecular docking of (c) RAPA and (d) CAN binding to the FRB domain within mTOR. (e) Common binding site of the FRB domain 
within mTOR to RAPA and CAN. (f) Comparison of the predicted Gibbs free energy of RAPA and CAN binding to the FRB domain of mTOR $(n=5)$. Ctrl, untreated normal control; PA, PA (0.25 mM)-treated control group; PA+CAN, HL-1 treated with PA $(0.25 \mathrm{mM})$ and CAN $(2.5 \mu \mathrm{g} / \mathrm{ml})$. RAPA, rapamycin; CAN, canagliflozin. NS, no significant difference; FRB, FKBP-RAPA-binding.

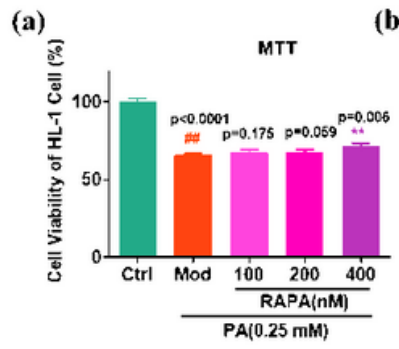

(f)

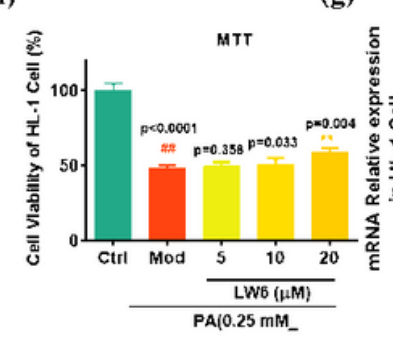

(k)

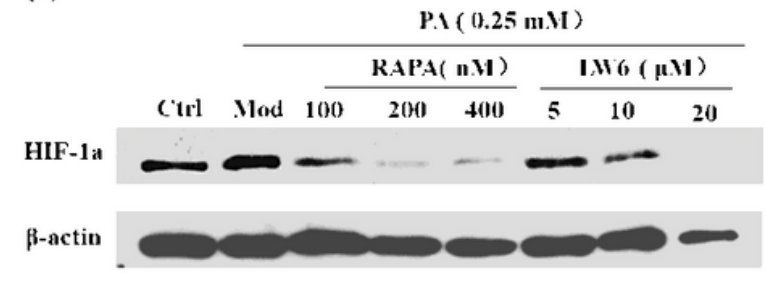

(c)

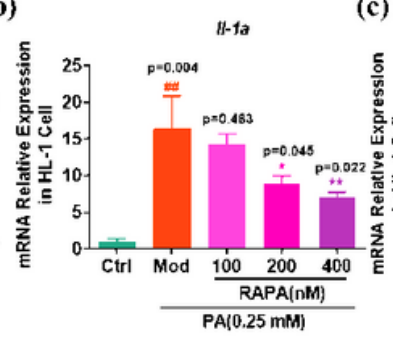

(h)
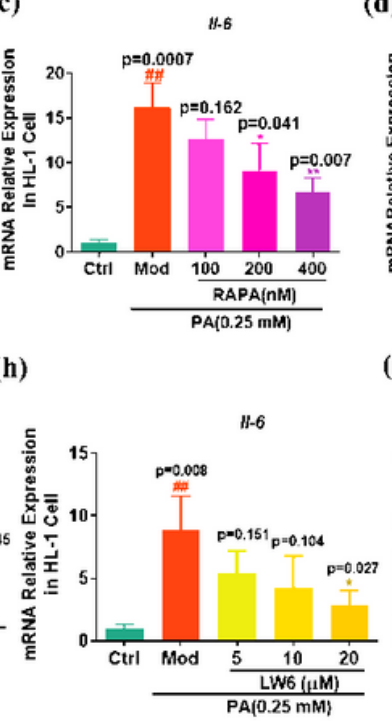

(d)

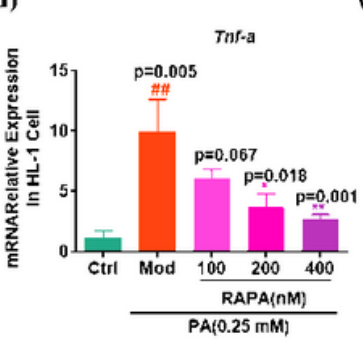

(i)
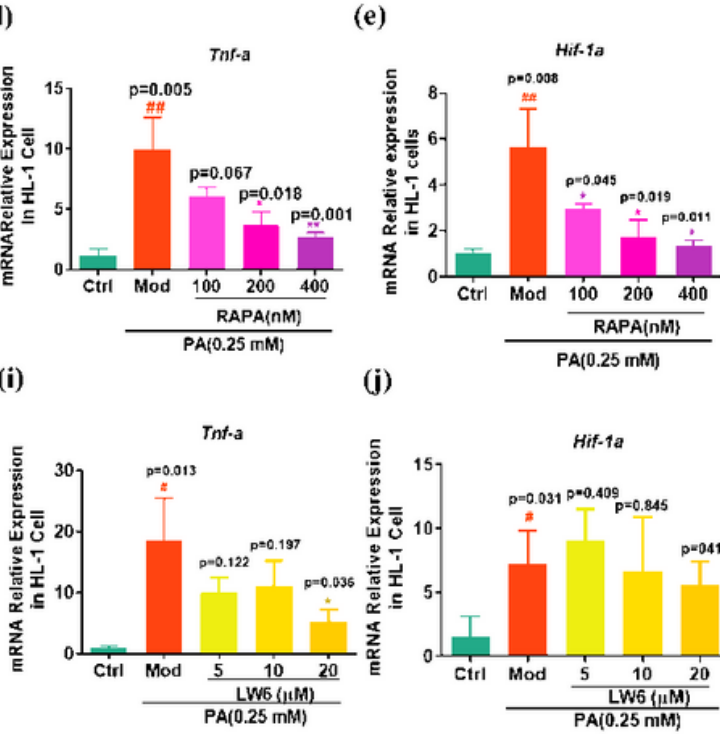

(j)

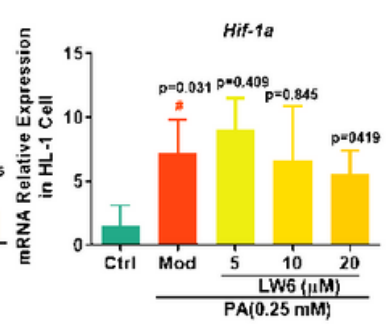

(l)

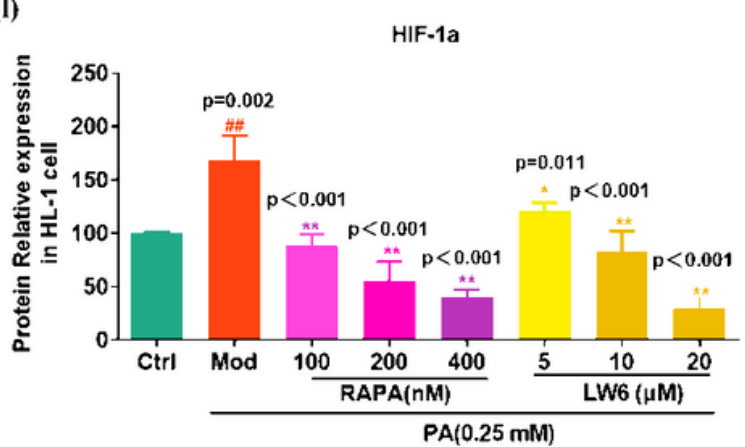

\section{Figure 6}

Protective effects of the mTOR inhibitor, RAPA and the HIF-1a inhibitor, LW6 on PA-induced HL-1 cell viability and inflammation. (a) Cell viability was detected using a MTT assay in the RAPA-treated HL-1 cells ( $n=3$ ). mRNA expression levels of (b) II-1a (c) II-6, (d) Tnf-a and (e) Hif-1a in the RAPA-treated HL-1 cells $(n=3)$. ( $f)$ Cell viability was detected using a MTT assay in the LW6-treated HL-1 cells $(n=3)$. mRNA expression levels of,(g) II-1a, (h) II-6, (i) Tnf-a and (j) Hif-1a in the LW6-treated HL-1 cells ( $n=3$ ). (I) protein expression levels of HIF-1a in RAPA and LW6 treated HL-1cells was determined using ImageJ software following western blot analysis $(k)$. The data are presented as the mean $\pm S D$. \#P<0.05 and \#\#P<0.01 vs. ctrl; ${ }^{*} P<0.05$ and ${ }^{*} \mathrm{P}<0.01$ vs. Mod. Ctrl, untreated normal control; Mod, $\mathrm{PA}(0.25 \mathrm{mM})$ treated control group; $100, \mathrm{HL}-1$ cells were treated with PA $(0.25 \mathrm{mM})$ and RAPA $(100 \mathrm{nM}) ; 200, \mathrm{HL}-1$ cells were treated with PA $(0.25 \mathrm{mM})$ and RAPA $(200 \mathrm{nM}) ; 400, \mathrm{HL}-1$ cells were treated with PA $(0.25 \mathrm{mM})$ and RAPA (400 $\mathrm{nM}) ; 5, \mathrm{HL}-1$ cells were treated with PA $(0.25 \mathrm{mM})$ and LW6 $(5 \mu \mathrm{M}) ; 10, \mathrm{HL}-1$ cells were treated with PA $(0.25 \mathrm{mM})$ and LW6 $(10 \mu \mathrm{M}) ; 20, \mathrm{HL}-1$ cells were treated with PA $(0.25 \mathrm{mM})$ and LW6 $(20 \mu \mathrm{M})$. 